\section{DNA fingerprinting on trial}

SIR-Two points arise from Lander's interesting Commentary "DNA fingerprinting on trial"'. First, Lander describes Lifecodes's use of a criterion for comparing measurements on two profiles whereby bands that are within 3 standard deviations (s.d.) of each other are deemed to match. The weaknesses of this kind of approach in the context of forensic science were first exposed by Lindley ${ }^{2}$ more than 10 years ago. An arbitrary cut-off, whereby a difference of $2.95 \mathrm{~s}$.ds results in positive evidence whereas one of $3.05 \mathrm{~s} . \mathrm{ds}$ leads to an exclusion, makes something of a lottery of a forensic comparison. Furthermore, to apply the criterion in isolation from the population data is artificial: whereas we may feel comfortable about reporting an exclusion at 3.05 s.ds when the bands being compared are at a very common region of the population distribution, the decision looks dubious when they are in a sparse region. There is another problem with the application of this criterion to restriction fragment length polymorphism analysis: because errors in band positions within a profile are highly correlated, to apply two independent 3 s.d. tests to the two bands is incorrect.

Lindley's solution, albeit to a different type of evidence, is to calculate the bayesian likelihood ratio. In this way, in the present context, the band measurements to be compared would be combined with the relevant population data in a function which will change smoothly from relatively large values, if bands are close, to progressively smaller values the further apart they are. Values of the likelihood ratio greater than one will support the explanation that the two profiles have the same origin, whereas values less than one will support the explanation that they have different origins. It is straightforward to handle correlated errors.

Second, Lander explains the process of using the frequencies of bands occurring in windows of arbitrarily selected size to estimate match probabilities. Deciding on the correct window width is problematical as we have discussed elsewhere ${ }^{3}$. We believe that it is preferable to estimate the underlying probability density function by kernel density estimation, in a manner similar to that used by Evett et al. ${ }^{4}$ in the analysis of another bivariate forensic problem.

Nature's Opinion' on the issues raised by Lander said "The underlying difficulty is that science and the law differ in temperament." We agree entirely. Forensic scientists continually strive to bridge this temperamental divide which means that forensic science has a unique disciplinary status. Statistical practices which are successful in other scientific arenas are not necessarily suitable within forensic science. Our approach to the analysis of data from a single locus, based on Lindley's principles ${ }^{2}$, has recently been outlined at an FBI symposium ${ }^{6}$.

I.W. EVETT

D.J. WERRETT

P. GILL

Home Office Forensic Science Service, Aldermaston, Reading,

Berks RG7 4PN, UK

J.S. BUCKLETON

Department of Scientific and Industrial

Research,

Mount Albert Research Centre,

Auckland, New Zealand

1. Lander, E.S. Nature 339, 501-505 (1989)

2. Lindley, D.V. Biometrika 64, 207-213 (1977)

3. Gill, P., Sullivan, K., Werrett, D.J. Hum. Genet., submitted. 4. Evett, I.W., Cage, P.E. \& Aitken, C.G.G. J. R. stat. Soc. (C) 36. 174-180 (1987).

5. Nature 339, 491-492 (1989)

6. Werrett, D.J., Gill P., Evett I.W., Lygo J.E. \& Sullivan, K.M. FBI Seminar on DNA Technology in Forensic Science (in the press).

\section{SN1987A pulses}

SIR-The reported 8-hour periodicity' in the emission from the pulsar in SN1987A may be due to the presence of a companion object ${ }^{1,2}$, but the companion would somehow have to have been created after the explosion. Long period modulations due to pulsations in the neutron star itself can be excluded ${ }^{2}$. Another possibility is that the 8-hour periodicity results from 'shaking' of magnetic field lines near the region of optical emission. For pulses at either the rotation frequency or the lowest radial vibrational mode $^{3}$ and nominal models of the neutron-star structure ${ }^{4.5}$, there is the possibility of exciting longperiod hydromagnetic modes which involve global oscillations of the neutron star with periods of about 8 hours. But such oscillations cannot account for the observed amplitude of the oscillation shift without being highly nonlinear in the associated displacements and magnetic field perturbations ${ }^{1}$. Most of the frequency variation, if it is real $^{3}$, probably results from another effect.

There is a long-period 'hydromagnetic inertial' mode for which magnetic tension is balanced by the Coriolis force in the rotating frame $^{5,15}$ (the Lense-Thirring effect modifies the quantitative details of this mode for realistic neutron-star models ${ }^{6}$ ). This mode occurs if the rotational speed at the star surface is small compared to the 'average' Alfvén speed through the star (oscillations of the superfluid vortex lattice yield even longer periods ${ }^{7}$ ). The associated shaking of the magnetic field lines in the emission region could affect the beaming of the pulses, and, hence, their intensity ${ }^{8,9}$, which could explain observed variations in the luminosity'.
For a constant density sphere with a constant, spin-aligned magnetic field, the oscillation frequency of the lowest (torsional) normal hydromagnetic inertial mode is ${ }^{10,11} \omega \simeq(8.9 / 2 \Omega)\left(V_{\mathrm{A}}^{2} / a^{2}\right)$ where $a$ is the radius of the oscillating sphere, $\Omega$ the spin frequency and $V_{\mathrm{A}}$ the average Alfvén speed.

The superconducting core protons are coupled to the magnetic field via the normal electrons, and the proton mass density is $\sim 0.05$ of the neutron mass density $^{12}$; if the more massive neutron core is coupled to the magnetic field via the protons and/or electrons on a timescale much less than 8 hours $^{12}$ then the Alfvén speed through the core will be smaller by a factor $\sim 4.5$ for the same magnetic field strength than if the coupling time were relatively long ${ }^{13}$.

With $\omega=2 \pi /(8 \mathrm{~h}), \Omega \simeq 1.2 \times 10^{4} \mathrm{~s}^{-1}$ and 'average' density $\sim 1.5 \times 10^{15} \mathrm{~g} \mathrm{~cm}^{-3}$ out to $\sim 7 \mathrm{~km}$ (from model $\mathrm{G}$ of ref. 6 ), we obtain a magnetic field $B \simeq 7.4 \times 10^{13} \mathrm{G}$. This value decreases to $\sim 1.6 \times 10^{13} \mathrm{G}$ if only the protons in the core provide the effective mass density. But the observed luminosity limits the magnetic field to $\sim 10^{9} \mathrm{G}$ (refs 1,3 ).

Such a small field may still be in agreement with a global oscillation. As the tilt angle of the magnetic field is increased with respect to the spin axis, components of the governing equation of motion tend to be decoupled for short wavelength oscillations $^{15}$. We can estimate a fundamental mode (Alfvén) frequency as $\omega \simeq \pi V_{\mathrm{A}} / a$. The implied field is then in agreement with the luminosity limit: $\sim 6.7 \times 10^{9} \mathrm{G}$ or $1.5 \times 10^{9} \mathrm{G}$ (ref. 16) (no neutron coupling).

For the oscillation to remain linear, the maximum angular displacement of the crust should be limited to $\sim 0.1$ turn $(\sim 0.6$ radians). Hence, for a torsional oscillation with a period of 8 hours, we require $\Delta \Omega \leqslant \sim 1.3 \times 10^{-4} \mathrm{~s}^{-1}$, which is smaller than the observed variation by a factor of about 70. This limit is within a factor of four of residuals found in a best fit to the data but is also within the range of uncertainty in the frequency itself ${ }^{1}$. For these modes to account for the observed frequency modulation, the magnetic field would be twisted through several complete revolutions. The frequency would then be much larger than implied by the linear treatment, but, it is not clear that the lattice structure of the magnetized vortices could be neglected in such an extreme case.

In the radial-vibration model ${ }^{3}$, rotational effects still dominate the propagation of hydromagnetic waves. The magnetic field values inferred above for a 0.1 -second period are $5.3 \times 10^{12} \mathrm{G}$ and $1.2 \times 10^{12} \mathrm{G}$ (again, depending on coupling in the core), consistent with the observed luminosity.

If the radial pulsation frequency is 